\title{
ACCRETION KINEMATICS THROUGH THE WARPED TRANSITION DISK IN HD 142527 FROM RESOLVED CO(6-5) OBSERVATIONS
}

\author{
S. Casassus ${ }^{1,2}$, S. Marino ${ }^{1,2}$, S. Pérez ${ }^{1,2}$, P. Roman ${ }^{2,3}$, A. Dunhill ${ }^{2,4}$, P. J. Armitage ${ }^{5}$ J. Cuadra $^{2,4}$, A. Wootten $^{6}$, \\ G. van der Plas ${ }^{1,2}$, L. Cieza ${ }^{2,7}$, Victor Moral ${ }^{2,3}$, V. Christiaens ${ }^{1,2}$, and Matías Montesinos ${ }^{1,2}$ \\ ${ }^{1}$ Departamento de Astronomía, Universidad de Chile, Casilla 36-D, Santiago, Chile \\ 2 Millennium Nucleus "Protoplanetary Disks," Chile; scasassus@u.uchile.cl \\ ${ }^{3}$ Center for Mathematical Modeling, Universidad de Chile, Av. Blanco Encalada 2120 Piso 7, Santiago, Chile \\ ${ }^{4}$ Instituto de Astrofísica, Pontificia Universidad Católica de Chile, 7820436 Macul, Santiago, Chile \\ 5 JILA, University of Colorado and NIST, UCB 440, Boulder, CO 80309, USA \\ ${ }^{6}$ National Radio Astronomy Observatory, 520 Edgemont Road, Charlottesville, VA 22903-2475, USA \\ ${ }^{7}$ Facultad de Ingeniería, Universidad Diego Portales, Av. Ejército 441, Santiago, Chile \\ Received 2015 May 28; accepted 2015 August 6; published 2015 September 24
}

\begin{abstract}
The finding of residual gas in the large central cavity of the HD 142527 disk motivates questions regarding the origin of its non-Keplerian kinematics and possible connections with planet formation. We aim to understand the physical structure that underlies the intra-cavity gaseous flows, guided by new molecular-line data in $\mathrm{CO}(6-5)$ with unprecedented angular resolutions. Given the warped structure inferred from the identification of scattered-light shadows cast on the outer disk, the kinematics are consistent, to first order, with axisymmetric accretion onto the inner disk occurring at all azimuths. A steady-state accretion profile, fixed at the stellar accretion rate, explains the depth of the cavity as traced in CO isotopologues. The abrupt warp and evidence for near free-fall radial flows in HD 142527 resemble theoretical models for disk tearing, which could be driven by the reported low-mass companion, whose orbit may be contained in the plane of the inner disk. The companion's high inclination with respect to the massive outer disk could drive Kozai oscillations over long timescales; high-eccentricity periods may perhaps account for the large cavity. While shadowing by the tilted disk could imprint an azimuthal modulation in the molecular-line maps, further observations are required to ascertain the significance of azimuthal structure in the density field inside the cavity of HD 142527.
\end{abstract}

Key words: planet-disk interactions - protoplanetary disks - stars: individual: (HD 142527)

\section{INTRODUCTION}

While in its first year of Early Science operations, the Atacama Large Millimeter Array (ALMA) already provided resolved sub-millimeter observations of circumstellar disks around young stars, where planet formation must occur. The pre-main-sequence star HD 142527 (F6me, at a distance of $\sim 145 \mathrm{pc}$ ) is particularly interesting because its disk is viewed close to face-on, and its protoplanetary cavity is amenable to scrutiny thanks to its record size (Fukagawa et al. 2006). Indeed, ALMA Cycle 0 observations of HD 142527 (Casassus et al. 2013c; Perez et al. 2015) revealed gaseous flows inside the dust-depleted central cavity, which is an expected feature of the formation of planetary systems (Dodson-Robinson \& Salyk 2011; Zhu et al. 2011). Here we complete this ALMA program with high-frequency molecular-line data that provide additional insight on the physical structures giving rise to the intra-cavity flows in HD 142527.

An obvious feature of the first ALMA observations of HD 142527 is the strong and centrally peaked $\mathrm{HCO}^{+}(4-3)$ emission, which is consistent with radial flows comparable in magnitude to Keplerian rotation (Casassus et al. 2013c). In this scenario, the mass transfer rate inferred from the $\mathrm{HCO}^{+}$ kinematics matches the stellar accretion rate. It is tempting to associate the $\mathrm{HCO}^{+}$kinematics with planet-induced accretion flows, i.e., gaseous streamers due to as yet undetected protoplanets inside the cavity. Such accretion kinematics should have counterparts in other molecular tracers, and indeed the velocity centroid map in $\mathrm{CO}(3-2)$ also hints at nonKeplerian flows (Casassus et al. 2013b, 2013c). However, the coarse Cycle 0 beam in $\mathrm{CO}(3-2)$, along with absorption by a diffuse CO screen along the line of sight to HD 142527 (Casassus et al. 2013a), prevent the adequate sampling of the intra-cavity kinematics. Rosenfeld et al. (2014) investigate the kinematic signatures of radial inflows and report free-fall radial velocities in $\mathrm{HD} 142527$ based on $\mathrm{CO}(3-2)$ and $\mathrm{HCO}^{+}(4-3)$, in addition to Keplerian rotation, but point out that a warped disk could also explain the observations (see also Hughes et al. 2009).

The new ALMA observations we present here, acquired in the $\mathrm{CO}(6-5)$ line, together with a well-determined inner disk orientation thanks to the identification of its shadows in scattered-light (Marino et al. 2015), allow us to conclude that the intra-cavity flows do correspond to fast stellocentric accretion occurring at all azimuths, at close to the maximum possible velocities for bound gas. As the radial velocity buildsup to near free-fall, the azimuthal component brakes. Section 2 presents the observational results of the intra-cavity gas kinematics. Section 3 introduces the parametric models we use to interpret the data and compares the models with the observations. Section 4 discusses implications of the inferred kinematics in the light of the recent theoretical discovery of "disk tearing" in strongly warped disks (Nixon et al. 2013). Section 5 summarizes our conclusions on the kinematics of stellocentric accretion in the cavity of HD 142527.

\section{OBSERVATIONS}

HD 142527 was observed with ALMA in band 9, at $\sim 700 \mathrm{GHz}$, during 2012 June and with baselines ranging from 

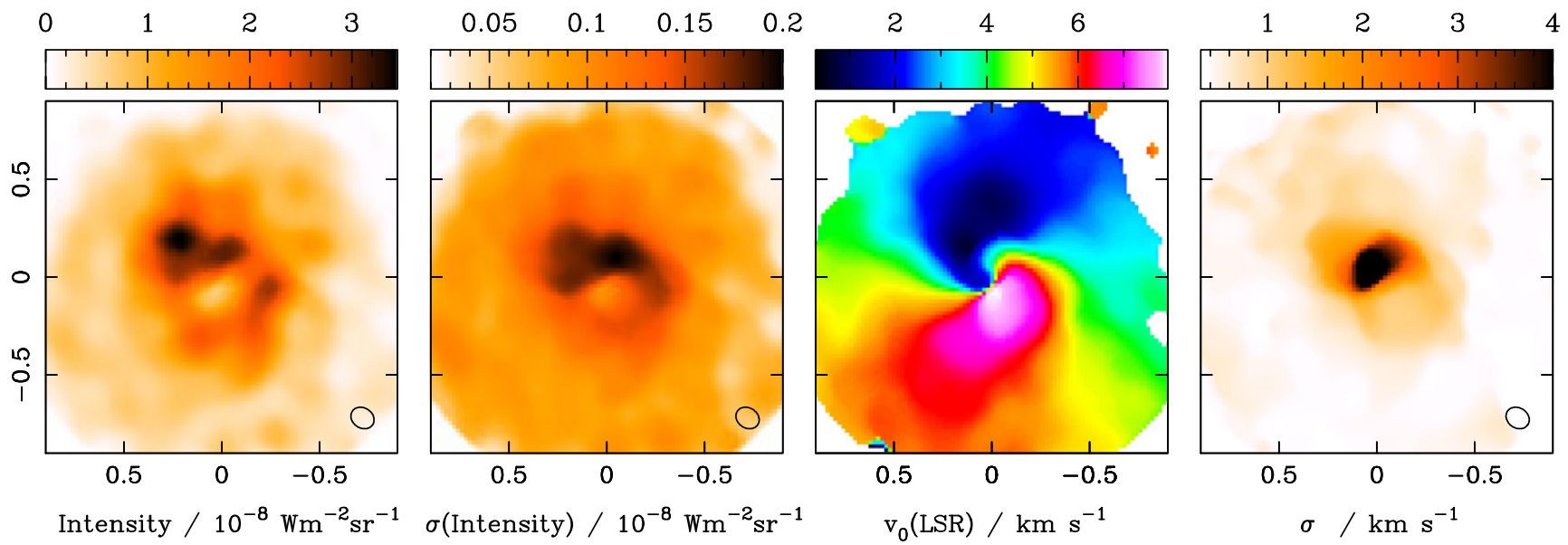

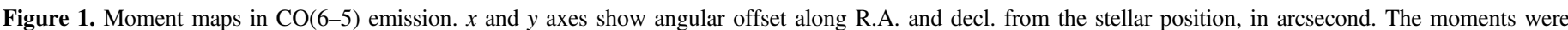

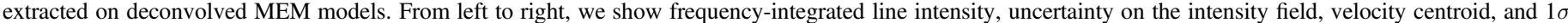
velocity dispersion.

$0.04 \mathrm{M} \lambda$ to $0.9 \mathrm{M} \lambda$. We refer to Appendix A.1 for an account of data reduction.

\subsection{Choice of Image-plane Representations of the Visibility Data}

Image synthesis was performed with a non-parametric leastsquares fit to the visibility data (see Appendix B); the resulting model images are labelled "MEM." These "deconvolved" images pick up finer details than the conventional "restored" maps, which are produced by convolving the MEM maps with an ellipical Gaussian called the "clean" beam, which is fit to the "dirty" beam (or point-spread function, PSF). With the addition of a dirty map of the model residuals, the restored maps allow a standard representation of the data, with a single and well defined angular resolution. However, the moments extracted on the "restored" maps (Appendix B) are affected by convolution with the clean beam, which enhances velocity dispersions by aligning different velocity components, with consequences on the intensity maps if extracting moments with Gaussian spectral fits (see Appendix B.3). We thus chose to base our study on the moment maps inferred from deconvolved (MEM) datacubes. A drawback of this choice is that since we are extrapolating power in the Fourier domain, the resolution of the MEM images is variable and depends on the image. Here the FWHM of the MEM PSF varies from 0 ". $062 \times 0$ ". 051 for a bright point source, up to $\sim 50 \%$ coarser for fainter extended emission (approximated by a collection of spikes).

\section{2. $C O(6-5)$ Kinematics}

The main properties of the continuum-subtracted $\mathrm{CO}(6-5)$ data are summarized by the moment maps shown in Figure 1. The $\mathrm{CO}(6-5)$ line intensity displays a central decrement so that the minimum intensity is a factor of $\sim 0.25 \pm 0.03$ times lower than the average intensity in the brighter ring at a radius of 0.22 . This central decrement is probably related to photodissociation of $\mathrm{CO}$, so that the very central $\mathrm{CO}(6-5)$ could be rarefied and broad enough to be optically thin. The more extended emission in $\mathrm{CO}(6-5)$ seems to be ascribed to the dust-depleted cavity, with a sharp drop at $\sim 1$ arcsec, corresponding to the radius of the Bright crescent-shaped outer ring seen in the continuum. This sharp drop is absent in $\mathrm{CO}(3-2)$, which extends well outside the cavity, so that the $\mathrm{CO}$ temperature outside the cavity is probably not high enough to excite $J=6$.

The disk position angle (PA) is defined as the intersection of the plane of a disk with the plane of the sky. For Keplerian rotation in disks with axial symmetry, the velocity centroid maps bear reflection symmetry about the disk PA, and the highest Doppler-shifted emission toward the blue and red are aligned along the PA. In Figure 1, the velocity centroid is more complex than the usual butterfly pattern. It is as if the velocity field had been "twisted" anti-clockwise, which would happen if the disk PA rotated with stellocentric radius $r$, from $-20^{\circ}$ east of north outside $r \sim 0$." 6 , to $\sim+60^{\circ}$ at $r \sim 0$ !" 25 , coincident with the extremes in velocity centroid. Inside 0 !" 1 , the PA appears to rotate further and reaches $110 \pm 10^{\circ}$. However, the scattered light shadows from the inner disk show that its PA is $-8 \pm 5^{\circ}$ (Marino et al. 2015).

The $\mathrm{CO}(6-5)$ velocity dispersion reaches very high values, especially near the star. Velocity dispersion values above $4 \mathrm{~km} \mathrm{~s}^{-1}$ are found in the unresolved central beam; the bulk of this dispersion is probably due to flow kinematics. However, values of about $2 \mathrm{~km} \mathrm{~s}^{-1}$ are also found away from the central beam, which persist after deconvolution. For instance, in Figure 1 the $1 \sigma$ velocity dispersion in the restored maps, $\sigma_{\text {rest. }}$, measured at an offset of $\boldsymbol{P}=(+0 ! 2,+0 ! 2)$, is $2.01 \mathrm{~km} \mathrm{~s}^{-1}$, while the $1 \sigma$ dispersion extracted from the deconvolved datacubes, $\sigma_{\mathrm{mem}}$, still shows a dispersion of $1.78 \mathrm{~km} \mathrm{~s}^{-1}$. Further tests on the suitability of the deconvolved images as measures of the intrinsic line width are provided in Appendix C.

Beyond 0."1 from the nominal stellar position, the line profiles appear to be singly peaked, as illustrated in Figure 2. If due to thermal broadening, the $\mathrm{CO}$ velocity distribution corresponds to temperatures $T=28 m_{p} \sigma^{2} / k$ in excess of $5000 \mathrm{~K}$, as far out as 0.15 from the star. Another mechanism for producing the observed line widths is microscopic turbulence. There are, however, hints of line asymmetry, such as shoulders or lack of low-level Gaussian wings, which suggest the presence of multiple velocity flows stratified along the line of sight. However, the significance of these spectral features is not high enough to motivate detailed interpretation.

Integrated line profiles for $\mathrm{CO}(6-5)$ are shown in Figure 3. The total line flux extracted from the restored datacube, using 

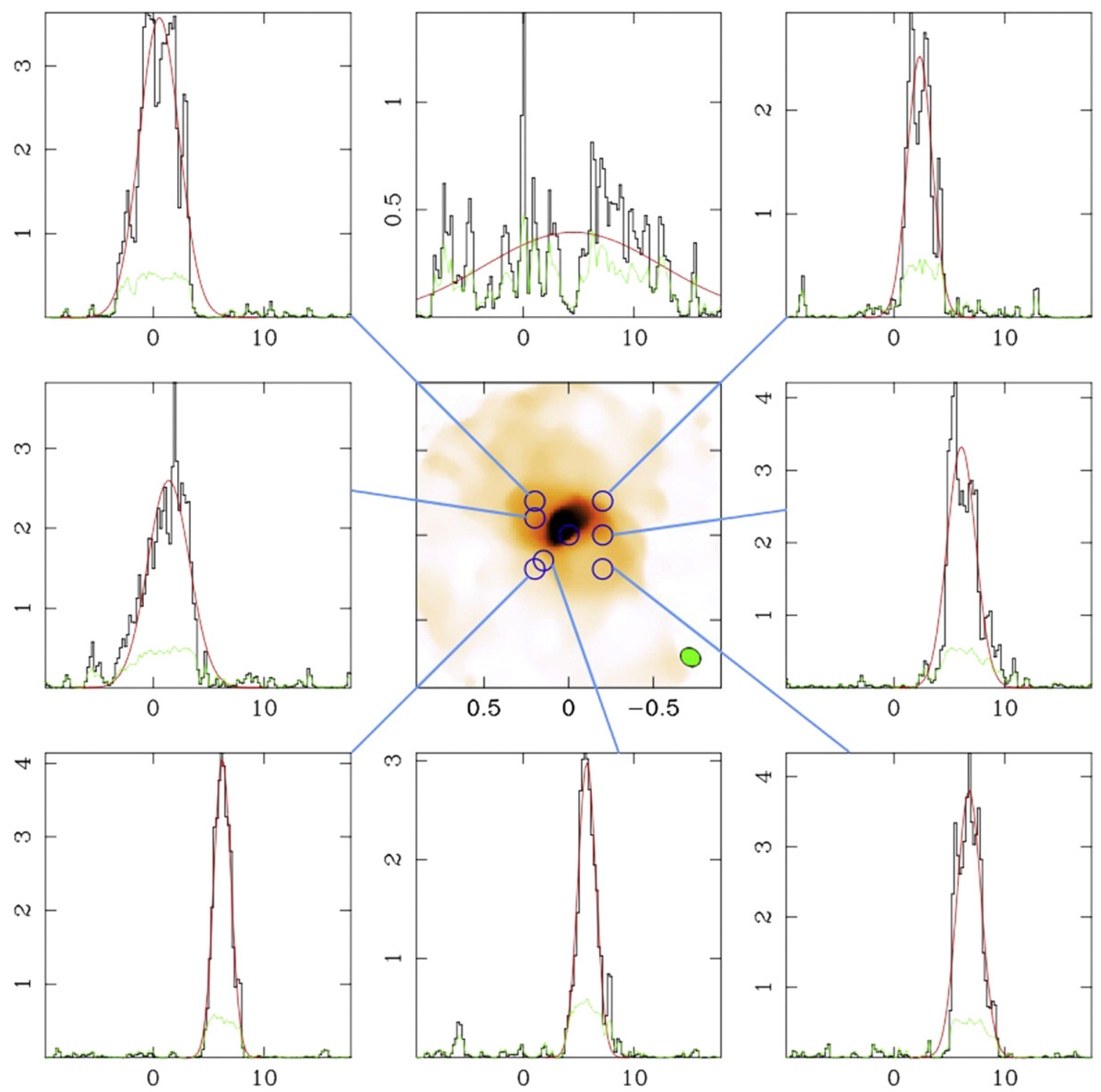

Figure 2. Example $\mathrm{CO}(6-5)$ line profiles. We select eight locations near the star to illustrate the line profiles obtained in the deconvolved datacube. The central plot shows $\sigma_{\mathrm{mem}}$ and is repeated from Figure 1. The spectra are extracted from single pixels in the deconvolved datacube. Solid black is used to plot specific intensity in $10^{11} \mathrm{Jy} \mathrm{sr}^{-1}$, along the $y$ axis, as a function of LSR velocity in the $x$ axis, in $\mathrm{km} \mathrm{s}^{-1}$. Solid green plots the $1 \sigma$ error on the specific intensity spectra, as estimated from Monte-Carlo runs (see Appendix B).

natural weights, and in a circular aperture with a radius of $2 . " 5$, is $70 \mathrm{Jy} \mathrm{km} \mathrm{s}^{-1}$. This value is fairly close to the flux density of $80 \mathrm{Jy} \mathrm{km} \mathrm{s}^{-1}$ reported by Casassus et al. (2013a) from APEX + CHAMP+ single-dish observations. The double-peaked CO (6-5) profiles obtained with ALMA are typical of Keplerian disks, and are also reminiscent of the CHAMP + spectrum, given its noise level. However, here the blueshifted peak appears to be fainter than the redshifted peak by $\sim 20 \%$. This line asymmetry is the opposite of that seen in ${ }^{12} \mathrm{CO}(2-1)$ (Perez et al. 2015), but is similar to $\mathrm{HCO}^{+}(4-3)$ (Casassus et al. 2013c) and the CO isotopologues (Perez et al. 2015). It is likely that the lower-J ${ }^{12} \mathrm{CO}$ is absorbed by the diffuse screen at redshifted velocities (Casassus et al. 2013a). The origin of the blueshifted decrement in species not affected by the diffuse molecular screen is probably related to the anti-correlation with the continuum: in continuum-subtracted maps, the line emission appears to be quenched under the crescent-shaped continuum (Casassus et al. 2013c; van der Plas et al. 2014;
Perez et al. 2015), at locations that correspond to blueshifted velocities. The pointing offset invoked by Casassus et al. (2013a) to account for the asymmetric CHAMP+ profile is probably unnecessary given the match in line flux with the interferometer $\mathrm{CO}(6-5)$ maps, and given the later finding of the molecular decrements at blueshifted velocities.

\subsection{Complementary Sub-millimeter Molecular-line Data}

As mentioned in Section 1 the $\mathrm{HCO}^{+}(4-3)$ and $\mathrm{CO}(3-2)$ data previously described in the literature also display deviations from Keplerian rotation with a constant disk orientation. Although the outer disk follows Keplerian rotation with a PA of $-20^{\circ}$, inside the cavity the PA joining regions of opposite velocities ${ }^{8}$ rotates with stellocentric distance resulting in "twisted" kinematics (Casassus et al. 2013b). The high

8 Relative to systemic. 

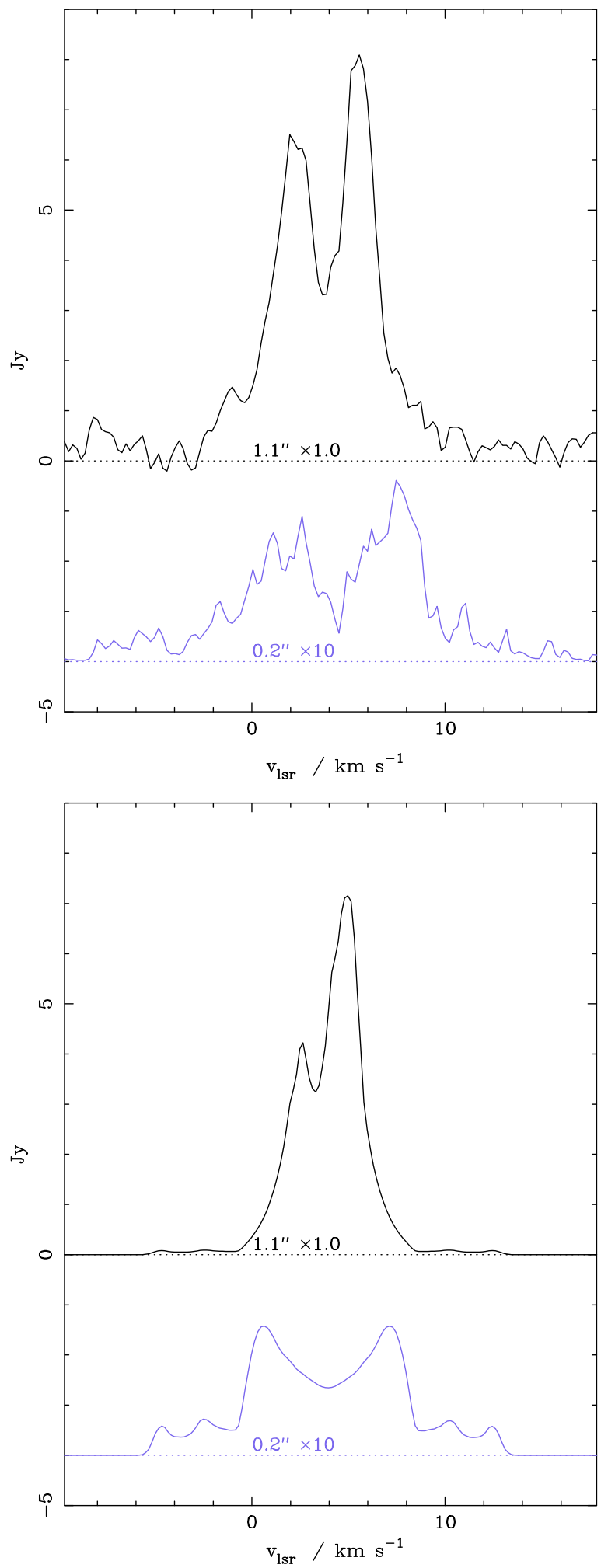

Figure 3. Line profiles in $\mathrm{CO}(6-5)$. Top: observed profile, extracted on the restored datacube in natural weights, using aperture photometry inside a 1 !" 1 radius (solid black), and also in the uvmem model with a 0.2 radius (in solid blue, magnified by a factor of 10 and offset for clarity). The horizontal dotted lines indicate the origins of flux density for each extraction. Bottom: corresponding line profiles extracted on the radiative transfer predictions described in Section 3. The wiggles in the line wings have counterparts in the observations - this may be better appreciated by inspection of the channel maps in Appendix A.1. velocity ${ }^{9}$ blue and redshifted $\mathrm{HCO}^{+}$are offset by 0.2 arcsec, with a PA of $\sim 60^{\circ}$ (Casassus et al. 2013c), while the outer disk PA is at approximately $-20^{\circ}$. Low-velocity filamentary $\mathrm{HCO}^{+}(4-3)$, within $0.8 \mathrm{~km} \mathrm{~s}^{-1}$ of systemic, connects the outer disk to the east with the central broad-velocity $\mathrm{HCO}^{+}$ (Casassus et al. 2013c), along a PA that coincides with that of the blueshifted signal. A similar but fainter filament may connect with the western outer disk. Interestingly, the peak $\mathrm{CO}$ (3-2) intensity is offset from the star, again toward the same direction as the blueshifted $\mathrm{HCO}^{+}$. The PA joining the blueand redshifted velocities is thus $\sim 60^{\circ}$ and common for $\mathrm{CO}(3-$ 2) and $\mathrm{HCO}^{+}$, and approximately orthogonal to the PA of the outer disk.

Such kinematic features, seen as extremes in $\mathrm{HCO}^{+}$, and probably also in $\mathrm{CO}(3-2)$, are expected in the framework of an additional radial velocity component directed toward the star. In order to impact the observed kinematics, the additional radial velocity component must be comparable in magnitude to the rotation velocity (Casassus et al. 2013b), so close to free-fall (Rosenfeld et al. 2014).

Figure 4 provides a summary of the data in each transition. It is apparent that the coarser beam in band 7, compared to band 9 , prevents a detailed study of the kinematics in the central 0 ". 3 . We also see in the deconvolved maps that attempts to trace the $\mathrm{CO}(3-2)$ kinematics inside the cavity are thwarted by the absorption from the intervening diffuse cloud in the line of sight to HD 142527 (e.g., Casassus et al. 2013a). Once convolved with the clean beam, the regions affected by the intervening $\mathrm{CO}$ cloud are less conspicuous and may lead to an accidental interpolation of the velocity field (as in Rosenfeld et al. 2014). Due to this intervening absorption, the PA in CO (3-2) as a function of radius does not appear to rotate beyond $30^{\circ}-40^{\circ}$ east of north, or $\lesssim 60^{\circ}$ relative to the outer disk. This shift is close to the value of $54^{\circ}$ obtained when the radial velocity component scales as $\sqrt{2}$ times the azimuthal velocity component (i.e., for a radial component close to free-fall).

\section{MODELING}

\subsection{Parametric Modeling}

In order to reproduce the intra-cavity kinematics, we developed on the accretion flows proposed to account for the $\mathrm{HCO}^{+}$ observations (Casassus et al. 2013c), while also taking into account the warp implied by the scattered-light shadows (Marino et al. 2015). We used a parametric model for HD 142527 that fits the spectral energy distribution (see Marino et al. 2015, for a detailed description), with the addition of a radial velocity component corresponding to stellocentric accretion. Emergent specific intensity fields were predicted by transferring the stellar radiation through the model circumstellar material with the RADMC3D ${ }^{10}$ package (version 0.39, Dullemond et al. 2015).

The essential elements of our model are depicted in Figure 5. The warp is implemented by rotating the disk as a function of stellocentric radius. The orientation of the outer disk, with a fixed inclination $i=160^{\circ}$ and PA $=-20^{\circ}$ beyond $r_{\text {orient_out }}=23 \mathrm{AU}$ (in spherical coordinates), is linked linearly at $r_{\text {orient_in }}=20 \mathrm{AU}$ with a non-coplanar inner disk, at a relative inclination of $70^{\circ}$ and at $\mathrm{PA}=-8^{\circ}$ (absolute east of north). The orientation of the inner disk is such that its

\footnotetext{
9 With velocities corresponding to $v_{\mathrm{lsr}} / \mathrm{km} \mathrm{s}^{-1} \in[-2.8,3.2]$ and $\in[4.8,8.8]$, while the systemic velocity is at $v_{\mathrm{lsr}}=+3.8 \pm 0.1 \mathrm{~km} \mathrm{~s}^{-1}$.

${ }^{10}$ http://www.ita.uni-heidelberg.de/dullemond/software/radmc-3d/
} 

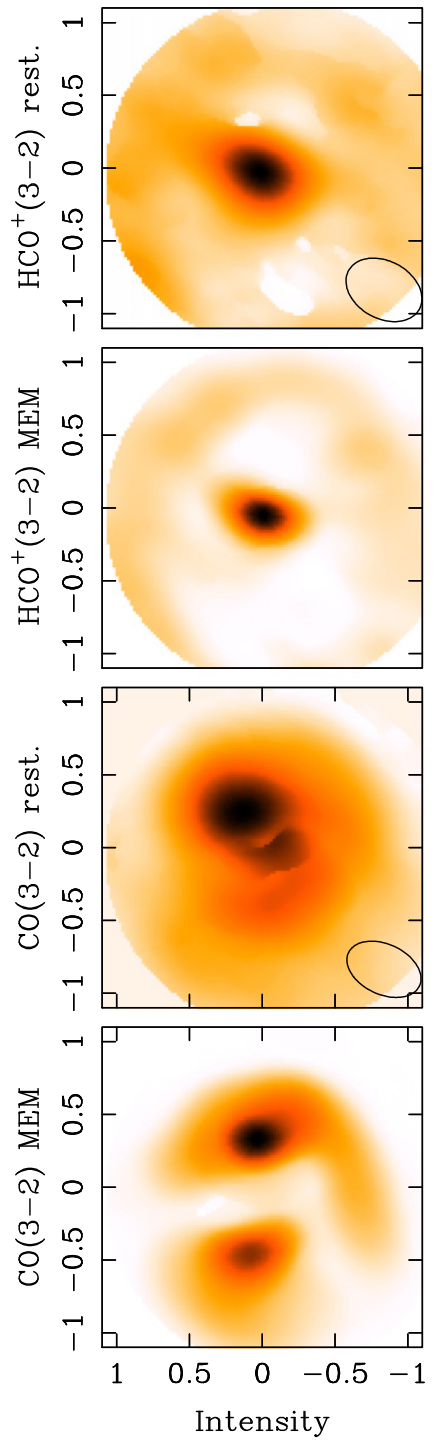
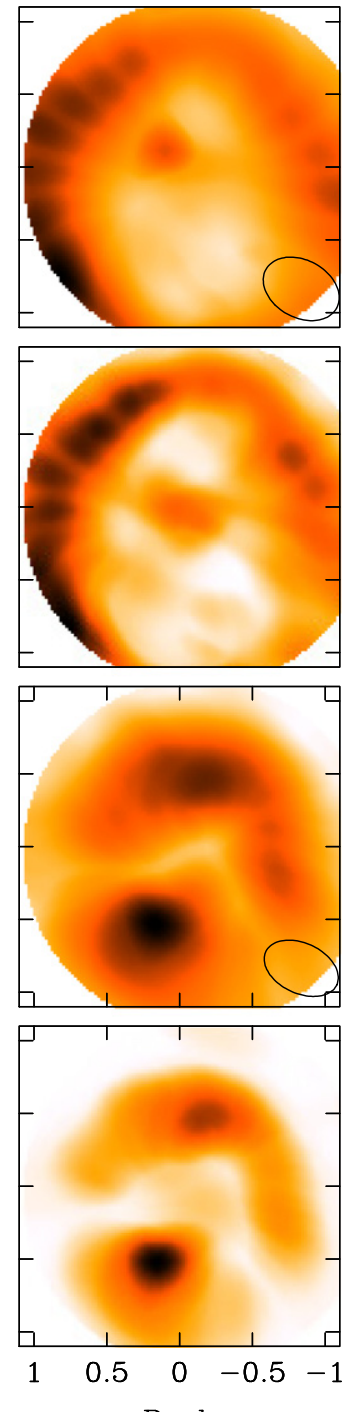
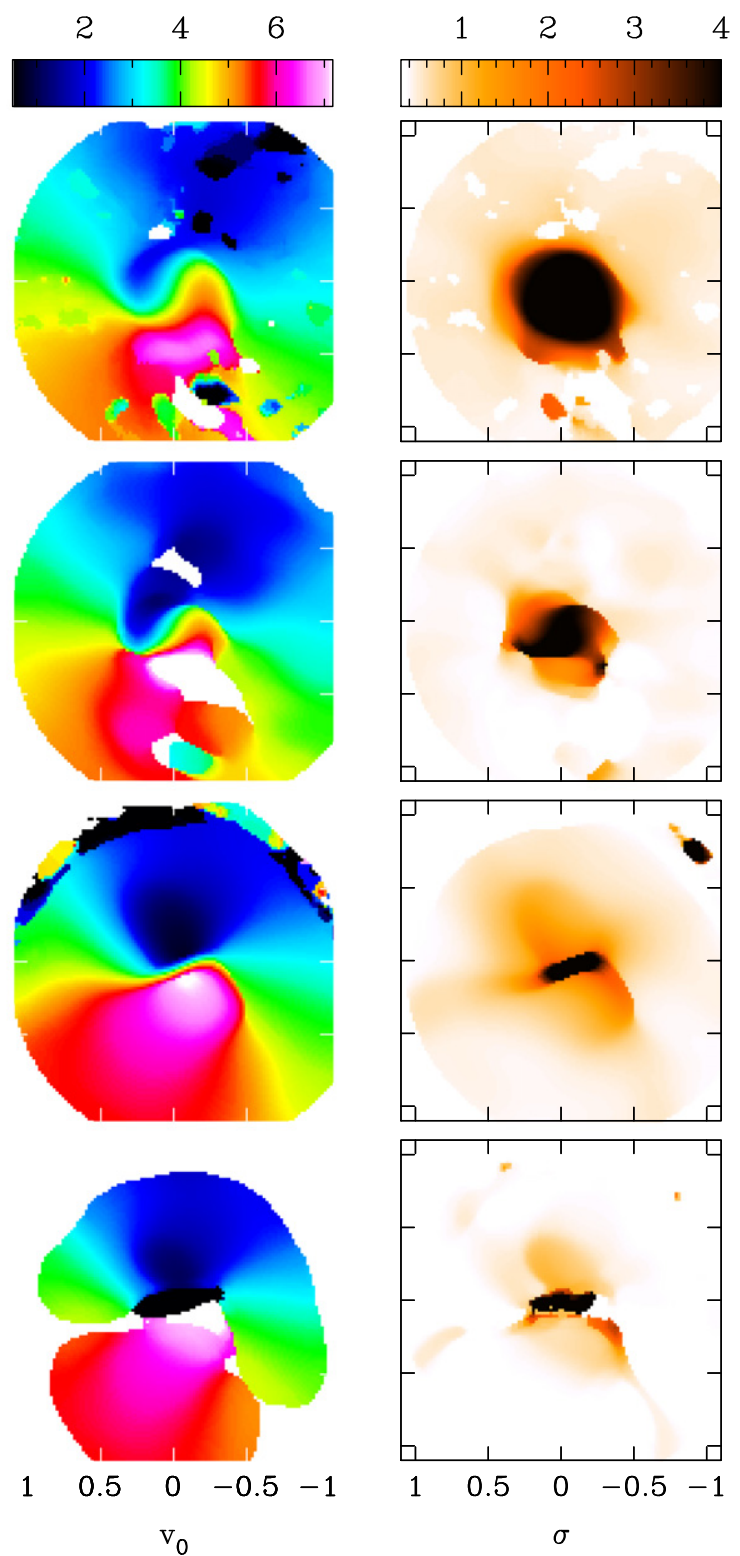

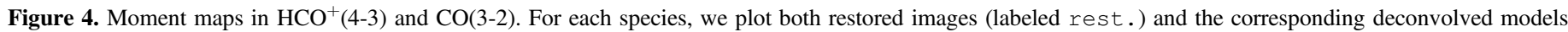

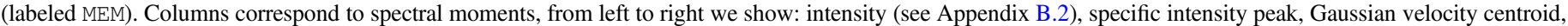
and Gaussian $1 \sigma$ velocity width.

inclination relative to the plane of the sky is $i \sim 1333^{\circ} 3$, so that the eastern side is near, while east is far for the outer disk. ${ }^{11}$ The inner disk probably contains the orbit of the low-mass companion reported by Biller et al. (2012) and Close et al. (2014).

Radial cuts illustrating our choice of velocity and surface density profiles are shown in Figure 6 inside the cavity. We caution that this parametric model is meant to help the interpretation of the data, and is not derived from a systematic search in parameter space. It is not a dynamically consistent model. Along with the warp, we also add a radial velocity component that accounts for accretion. We connect zero accretion at $\sim 140 \mathrm{AU}$ to free-fall at $30 \mathrm{AU}$, with a cubic profile. The free fall halts at 23.3 AU. The tilted inner disk is assumed to be in pure Keplerian rotation, so that inside $20 \mathrm{AU}$

\footnotetext{
${ }^{11}$ In other words, the parallactic angle of the normal to the disks is rotated by $168^{\circ}$.
}

we set zero accretion (a build-up of accreted mass onto the inner disk could then trickle to feed pole-on stellar accretion). The total surface density profile in the gap follows from the assumption of steady-state accretion:

$$
\Sigma(r)=\frac{d M_{\star}}{d t} /\left(2 \pi r v_{r}\right),
$$

as a function of spherical polar radius $r$, where $v_{r}$ is the radial velocity component,

$$
\begin{aligned}
& v_{r}=0 \text { if } r<r_{g 1}, \text { with } r_{g 1}=20 \mathrm{AU}, \text { or } \\
& v_{r}=-0.1 v_{\mathrm{ff}} \text { if } r<r_{\mathrm{stop}}, \text { with } r_{\mathrm{stop}}=23.3 \mathrm{AU}, \text { or } \\
& v_{r}=-0.9 v_{\mathrm{ff}} \text { if } r<r_{\mathrm{ff}}, \text { with } r_{\mathrm{ff}}=30 \mathrm{AU}, \text { or } \\
& v_{r}=-0.9 v_{\mathrm{ff}} \times\left[\frac{\left(f r_{g 2}-r\right)}{\left(f r_{g 2}-r_{g 1}\right)}\right]^{3} \text { if } r<r_{g 2},
\end{aligned}
$$




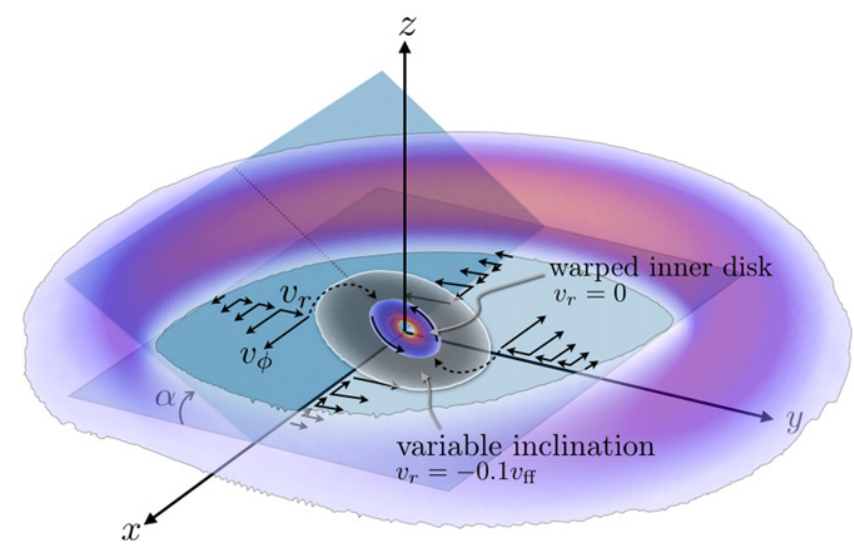

Figure 5. Illustration of the key elements of the parametric model that we propose to explain the $\mathrm{CO}(6-5)$ velocity field in HD 142527 . The crescent in hues of red and purple represents the distribution of millimeter-sized grains. The solid arrows trace the velocity field. Inside the warp that connects the two disk inclination, material flows along the dashed and curved arrows.

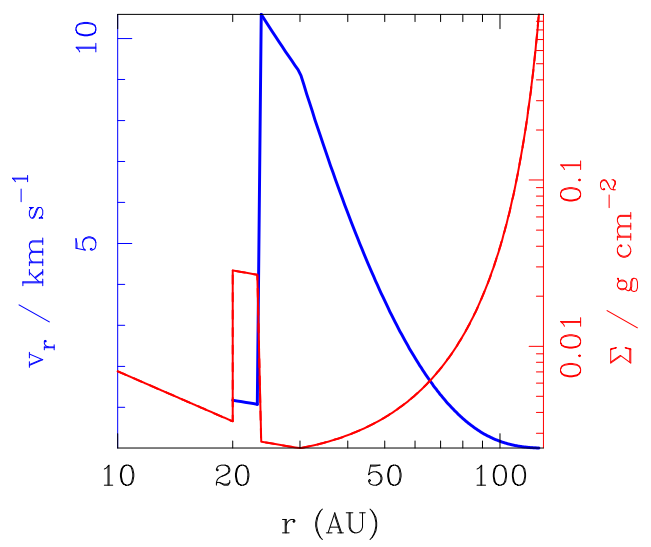

Figure 6. Radial cuts for the surface density profiles (red) and magnitude of the radial velocity component (blue). The $\mathrm{CO}$ abundance is modulated with a Gaussian taper inside $30 \mathrm{AU}$, to account for photodissociation.

with $r_{g 2}=130 \mathrm{AU}$, and where $f=1.1$ is a factor to avoid divergence at $r_{g 2}$, and

$$
v_{\mathrm{ff}}=\sqrt{2 G M_{\star} \times\left(\frac{1}{r}-\frac{1}{f r_{g 2}}\right)} .
$$

We use a stellar accretion rate of $d M_{\star} / d t=10^{-7} M_{\odot}$ (Garcia Lopez et al. 2006). The stellar mass is set at $1.8 M_{\odot}$; higher masses produce higher velocities inside the cavity than observed.

The orientation of the disk is parametrized in spherical coordinates, where the vertical axis $\hat{z}^{\prime}$ is the normal to the local disk plane, and where the origin of azimuth $\phi^{\prime}$ is the local line of nodes (i.e., the disk PA as a function $\boldsymbol{r}$ ). The azimuthal velocity $v_{\phi^{\prime}}$ is set to Keplerian rotation $v_{\phi^{\prime}}=v_{\mathrm{K}}$, except in regions where $\left|v_{r}\right|>0$, where we assumed that the specific energy density was close to 0 , so that $v_{\phi}=\sqrt{v_{\mathrm{ff}}^{2}-v_{r}^{2}}$ (neglecting the internal energy).

In the warp itself, between $r_{\text {orient_in }}=20 \mathrm{AU}$ and $r_{\text {orient_out }}=23 \mathrm{AU}$, material continuously connects both orientations. We investigated a range of values for the vertical velocity component in the warp and obtained a closer match to



Figure 7. Intensity maps (moments 0 ) extracted from the RADMC3D predictions, after smoothing to the resolution of the deconvolved $\mathrm{CO}(6-5)$ datacubes.

the observations with relatively fast velocities, $v_{\theta^{\prime}}=v_{\text {warp }}=$ $v_{\mathrm{K}} \sin \left(\phi^{\prime}\right)$ (see Section 3.2.2).

The temperature profile we adopted is $T=70 \mathrm{~K}$ if $r<30 \mathrm{AU}$, or

$$
T(r)=70\left(\frac{r}{30 \mathrm{AU}}\right)^{-0.5} \mathrm{~K}, \text { if } r>30 \mathrm{AU},
$$

which allows us to approximately reproduce the $\mathrm{CO}(6-5)$ line profile. Equation (2) is probably not the best representation of the actual $\mathrm{CO}$ temperature field. It could be improved by adding structure, especially in the outer disk, where the gas temperatures probably flatten out. Equation (2) results in gas temperatures slightly lower than the dust temperatures in the outer disk, which leads to negatives in continuum-subtracted data. These absorption features appear to be necessary to account for the decrements seen in the molecular-line in the outer disk, and which are discussed in a companion paper (Casassus et al. 2015, see also Section 3.2.1). These temperatures are also well below the dust temperatures in the closer-in regions. If we impose thermal equilibrium between both solid and gas phases, the predicted flux densities soar an order of magnitude above the observations.

Close to the star, $\mathrm{CO}$ is probably photodissociated. The line intensity of $\mathrm{CO}(6-5)$ appears to display a decrement inside 0 "! 2, which we accounted for with a Gaussian taper of the gas abundance inside $30 \mathrm{AU}$, with a $1 \sigma$ width of $5 \mathrm{AU}$.

\subsection{Radiative Transfer Predictions}

\subsubsection{Adopted Abundance Fields and Depth of the Cavity}

We adopted a nominal $\mathrm{H}_{2} /{ }^{12} \mathrm{CO}$ ratio $X_{\mathrm{CO}}=10^{-4}$, and the following molecular abundances relative to $\mathrm{H}_{2}$ (no selective photodissociation was required). For ${ }^{13} \mathrm{CO}$, we used the ISM isotopic abundance for ${ }^{12} \mathrm{C} /{ }^{13} \mathrm{C}$ of 76 (Stahl et al. 2008), 

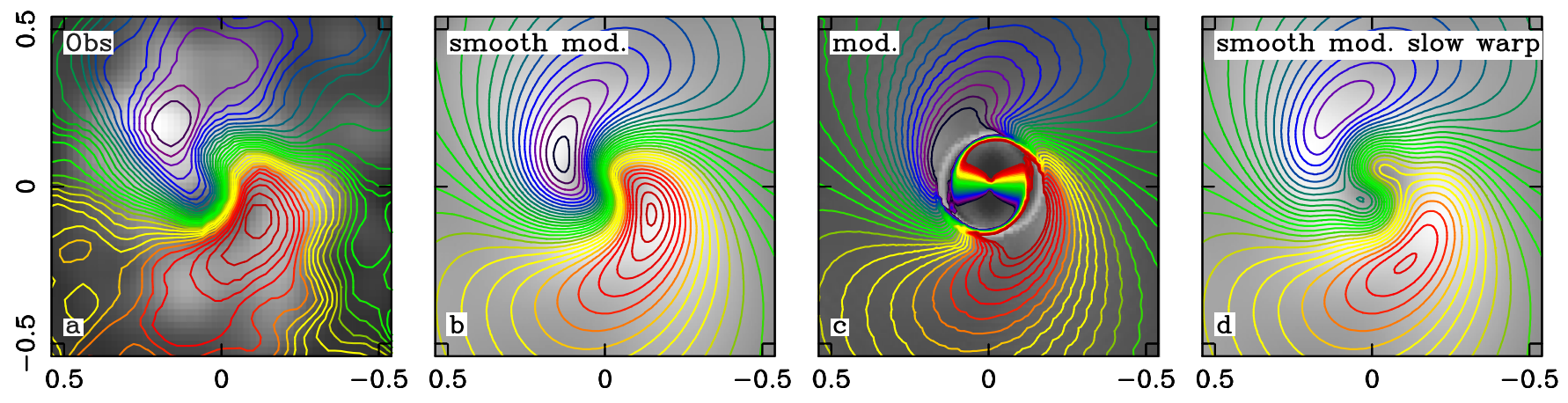

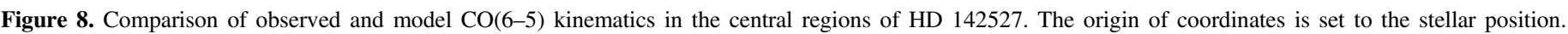

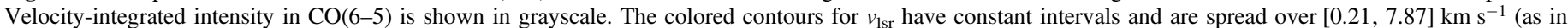



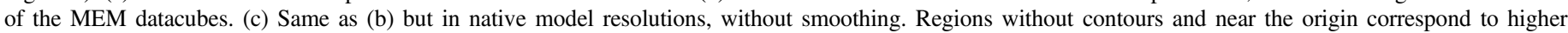
velocities. (d) Same as (b) but with a slow velocity component perpendicular to the disk plane ( $v_{\text {warp }}$ in the text).

multiplied by $X_{\mathrm{CO}}$. For $\mathrm{C}^{18} \mathrm{O}$, we proceeded as for ${ }^{13} \mathrm{CO}$, but with an ISM isotopologue abundance for $\mathrm{C}^{18} \mathrm{O} /{ }^{12} \mathrm{CO}$ of 500 (Wilson \& Rood 1994). For $\mathrm{HCO}^{+}$, we used a default value for the $\mathrm{HCO}^{+}$abundance relative to $\mathrm{H}_{2}$ of $10^{-9}$ (somewhat lower than observed in molecular cloud cores and comparable to values inferred in other disks, Wootten et al. 1979; Teague et al. 2015).

After smoothing to the resolution of the deconvolved models, Figure 7 shows that the accreting warped disk model qualitatively reproduces the basic properties of the cavity seen in the available molecular tracers. The model is in agreement with the non-detection of ${ }^{13} \mathrm{CO}$ and $\mathrm{C}^{18} \mathrm{O}$ inside the gap. Both in band 6 (Perez et al. 2015) and band 7 (Fukagawa et al. 2013), the ${ }^{13} \mathrm{CO}$ and $\mathrm{C}^{18} \mathrm{O}$ datacubes do not show any sign of either twisted kinematics, a central peak, or of the gap-crossing filaments. The CO isotopologue data display plain Keplerian rotation, without signal inside $\sim 90$ AU.

The decrements under the crescent-shaped continuum, previously reported by Casassus et al. (2013c), are a natural feature of the predictions. In continuum-subtracted images, and with our gas temperature prescription, the gas effectively acts as a cool foreground (Casassus et al. 2015).

\subsubsection{Central Beam}

The warp inside the cavity of HD 142527, i.e., the continuous change in inclination at some specific radius, should significantly impact the observed kinematics. For instance, given the orientation implied by the scattered light shadows (Marino et al. 2015), the blueshifted flows to the north should suddenly shift to red as the inclination changes sign, at the radius where the far side becomes the near side. Interestingly, as illustrated in finer detail in Figure 8(a), the observed centroid map indeed has structure very close to the central star, well inside the central beam. Within $\sim 0$ ". 25 , the "twist" in PA, i.e., the rotation of the axis joining blue- and redshifted velocities, appears to dwell at $\sim 40^{\circ}$, and thus is offset by $\sim 60^{\circ}$ from the outer disk PA, as expected for a radial velocity component that has reached close to free-fall values while also keeping in near-Keplerian rotation. Indeed, at a given stellocentric radius, in the presence of a radial velocity component that is $\sqrt{2}$ times the azimuthal component, the peak line-of-sight velocity is offset from the disk PA by $\sim 54^{\circ} .6$. However, inside 0 ." 1 , the PA joining blue and redshifted velocities appears to reach $110 \pm 10^{\circ}$.
The extreme in PA rotation in the inner 0 ". 1 cannot be accounted for by free-falling material that is also close to Keplerian rotation. This can instead be understood as the halt of free-fall inside a warped structure, as the blueshifted flows to the north-west $(11 \mathrm{hr})$ suddenly Doppler-flip into red closer to the star, where the inclination crosses the plane of the sky (the far side turns near). After smoothing to the resolution of the deconvolved MEM datacubes, we obtain an approximate match to the velocity field for an abrupt warp connecting the inner disk at $20 \mathrm{AU}$ with the orientation of the outer disk at $23 \mathrm{AU}$, as shown in Figure 8(b). The warp orientation must align to that of the inner disk as far out as $20 \mathrm{AU}$, to account for the shift in declination at systemic velocities, which covers almost 0 ". 2 when crossing the stellar position.

In a continuous warp, material must flow from one plane to the other and be accelerated in the transition. Thus, inside a warp, there should be a velocity component orthogonal to the plane of the disk $v_{\text {warp }}$. This component is probably limited by the Keplerian velocity $v_{\mathrm{K}}$ around the central star. For instance with $v_{r}=0.1 v_{\text {ff }}$ and $v_{\theta}=v_{\text {warp }}=v_{\mathrm{K}}$ in spherical coordinates, the warp's radial width must be at least $\sim 3$ AU to connect one orientation to the other. Interestingly, the inclusion of $v_{\text {warp }}$ approximates better the observed velocity centroid, with fairly point-symmetric high velocity ridges connecting the cavity "twist" with the inner disk, corresponding to the loci of bluest and redest velocities in Figure 8(b). These ridges are missing in the model without $v_{\text {warp }}$ (Figure 8(d)).

In order to further illustrate the model, we have included a radiative transfer prediction if the parametric model was viewed closer to edge-on, rather than face-on. Figure 9 shows the equivalent of Figure 8, for the case where the disk has an inclination of $66^{\circ}$. Since our adopted temperature field results in gas temperatures cooler than the continuum in the outer disk, in these edge-on predictions, the molecular line moments taken on the continuum-subtracted data are more strongly affected by extended negatives. Therefore, for the close to edge-on predictions, we have chosen not to subtract the continuum.

\section{DISCUSSION}

\subsection{Ro-vibrational CO}

The CRIRES observations by Pontoppidan et al. (2011) assign bright rovib $\mathrm{CO}$ to an inner disk, with similar inclination as the outer disk but offset in PA, at $61 \pm 3^{\circ}$-thus, within uncertainties coincident with the direction of the high-velocity 

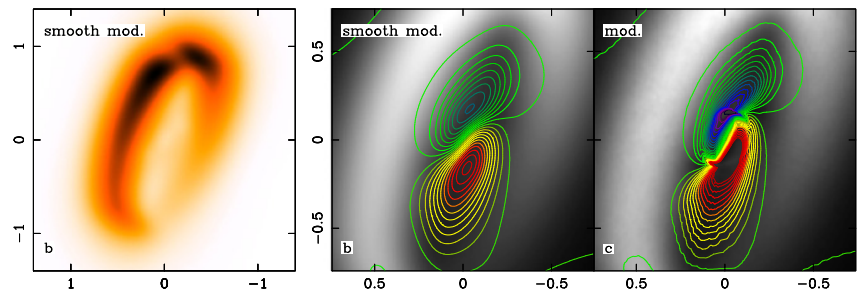

Figure 9. Radiative transfer prediction for the parametric disk model, if it were viewed at an inclination of $66^{\circ}$, and without continuum subtraction. (a) Moment 0 map, essentially tracing the continuum emission, and shown on a larger field to better convey the close to edge-on viewing angle. (b) Centroid of the velocity field extracted on the radiative transfer prediction, after smoothing to the resolution of the MEM datacubes. The colored contours for $v_{\mathrm{lsr}}$ have a constant interval and are spread over $[0.21,7.87] \mathrm{km} \mathrm{s}^{-1}$ (as in Figure 1). (c) Same as (b) but in native model resolutions, without smoothing. Regions without contours and near the origin correspond to higher velocities.

$\mathrm{HCO}^{+}$. The total line widths in $\mathrm{CO}(v=1-0)$ at $4.67 \mu \mathrm{m}$, at zero-intensity, are close to $\sim 40 \mathrm{~km} \mathrm{~s}^{-1}$ (Pontoppidan et al. 2011). With a spectroastrometric offset of only 0.2 AU, CO $(v=1-0)$ is either fairly uniform or else confined to the central few milliarcseconds.

We check the astrometric signal of a modified version of our disk model and find it to be in agreement with the spectroastrometric CRIRES observations. We take the predicted CO (6-5) emission and transform the intensity with an $1 / r^{\alpha}$ mask to mimic the $\mathrm{CO}$ ro-vibrational emission, which traces higher temperatures and emits closer to the star compared to the $\mathrm{CO}$ rotational lines. We manually add continuum emission scaled to reproduce a line to a continuum ratio of 1.1 , as in the observed rovibrational CO, and convolve with a 0". 192 FWHM Gaussian to mimic the PSF of the AO-assisted CRIRES observations. After superimposing a 0 !' 2 arcsec wide slit positioned at $150^{\circ}$, we recover a line profile and spectroastrometric signal similar in shape and magnitude to that reported by Pontoppidan et al. (2011) when using $\alpha \sim 2.5$.

\subsection{What About an Outflow?}

The orientation of the disk is well known. As explained in Sections 1 and 3.1, the shadows seen in scattered light imply that the inclination of the outer disk is such that the far side lies to the east. The eastern side being brighter in the mid-IR is also in support of this orientation (Fujiwara et al. 2006). In addition, several near-IR spirals have been found that are all consistently trailing (Fukagawa et al. 2006; Casassus et al. 2012; Canovas et al. 2013; Avenhaus et al. 2014), even in their radio extensions into the outer disk (Christiaens et al. 2014). Given this orientation, we can rule out an outflowing disk wind: the velocity component orthogonal to the disk would broaden the lines and preserve reflection symmetry about the outer disk PA, while the radial component in the plane of the disk would twist the kinematics in the opposite direction than observed.

\subsection{Origin of the Free-falling Velocities}

The radial velocity in a warped viscous disk is predicted to be greater than in a flat disk (Pringle 1992), but is not expected to exceed the sound speed $c_{s}=(h / r) v_{\mathrm{K}}$. Supersonic disk inflow is in principle possible in regions of the disk that lose angular momentum rapidly through a magnetized wind, rather than via internal viscous transport, though whether this can occur without equally rapid mass loss (for which there is no evidence in HD 142527) is not clear. Alternatively, the free-fall velocity signal could be a consequence of strong dynamical perturbations. If the low-mass companion detected in the system (Biller et al. 2012; Close et al. 2014) is misaligned to the outer disk in the same way as the inner disk, which is known to be inclined by $70^{\circ}$ (Marino et al. 2015), then an observable dynamical impact is highly likely. The alignment of the companion in the plane of the inner disk seems like a natural way to explain the inclination of the inner disk itself. Miranda \& Lai (2015) have recently shown that large offsets between the orbital planes of circumprimary disks and the plane of the binary can be stable for several megayears, longer than the expected lifetime of the disk. The origin of the companion and its possibly tilted orbit relative to the outer disk are to be searched for in the context of clustered star formation from a turbulent cloud, for instance through a late accretion episode (as investigated by Bate et al. 2010).

It has recently been shown that such strongly misaligned binary-disk systems can undergo a process dubbed disk tearing (Nixon et al. 2013; Doğan et al. 2015; Nealon et al. 2015), where nodal precession torques induced by the binary produce a warp at the inner edge of the disk. If the disk is unable to communicate the warp efficiently, then the disk will effectively break at some radius $R_{\text {break }}$. Annuli of gas then begin to tear off of the inner edge and can precess freely, undergoing selfinteraction and angular momentum cancellation causing a subsequent infall of gas driving very high accretion rates (Nixon et al. 2012). In the context of circumstellar disks, Facchini et al. $(2013,2014)$ show that in the thick-disk regime, where $\alpha<H / R$, an inner warp greater than $\sim 40^{\circ}$ will break the disk into distinct planes.

To date, the process of disk tearing has primarily been studied in the thin-disk regime appropriate for black hole accretion, where the dimensionless viscosity parameter is larger than the disk aspect ratio, i.e., $\alpha>H / R$ (Nixon et al. 2012, 2013). Nevertheless, it has also been shown to work in the thick-disk regime where $\alpha<H / R$ (Nealon et al. 2015), which is more likely to hold true for circumstellar disks like HD 142527.

It is possible to estimate a value for the radius $R_{\text {break }}$ at which disk tearing will occur (see Equation (A3) of Nixon et al. 2013) using the parameters of the disk and binary. With appropriate values for HD 142527, we expect that the disk could break at $R_{\text {break }} \sim a$, where $a$ is the binary semimajor axis. However, Nealon et al. (2015) found a consistent offset between the estimated minimum value and the true breaking radius in a series of smoothed particle hydrodynamics simulations, by a factor of two to three. Estimates of $a \sim 15 \mathrm{AU}$ are therefore entirely consistent with a breaking radius of $30 \mathrm{AU}$, as demanded by our model. However, because this is likely very sensitive to the parameters of the system, a more quantitative comparison of this scenario with the observed data is out of the scope of this paper, requiring the development of targeted numerical simulations.

We also note that while this scenario seems to adequately explain both the inner warp and the free-falling velocities, it does not explain the presence of the $\sim 130$ AU cavity in the system. This should not be surprising, given the large difference in scale between the warp and the cavity (and therefore different timescales associated with physical processes at these radii), but means that the model is not yet a complete description of the system. 


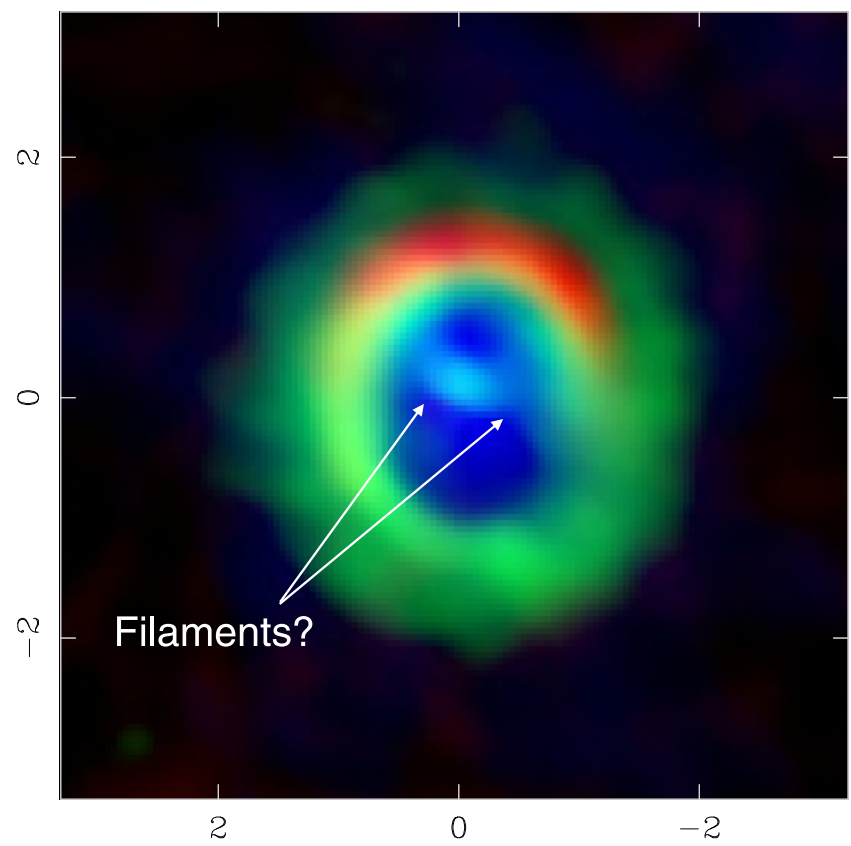

Figure 10. Summary of Cycle 0 band 7 observations, from MEM maps, with continuum in red, $\mathrm{HCO}^{+}(4-3)$ in green, and $\mathrm{CO}(3-2)$ in blue. $x$ and $y$ axes show offset from the star, in arcsecond. Velocities have been restricted to highlight the fainter structures seen in $\mathrm{HCO}^{+}$, which are otherwise dwarfed by the fast $\mathrm{HCO}^{+}$central emission.

\subsection{Long-term Kozai Oscillations and the Large Cavity}

The dynamical influence of the low-mass companion on its current orbit will not extend out to the $100 \mathrm{AU}$ scale needed to explain the large extent of the cavity. It is possible, however, that its orbit may have changed over time. The highly inclined orbit of the companion relative to that of the massive outer disk could trigger Kozai oscillations (Teyssandier et al. 2013), with a period of the order of $10^{5}$ year if the outer disk has a mass of $0.1 M_{\odot}$. A Kozai cycle would result in (damped) oscillations between the current inclined circular orbit, and a highly eccentric coplanar orbit, which would perturb the disk out to greater radii. Numerical simulations are needed to determine if such a cycle is possible in HD 142527.

\subsection{Non-axial Symmetry in the Cavity: Signature of Obscured Planets?}

Observationally, we cannot test directly for past coplanar eccentric orbits, as in the Kozai oscillations, but we can instead look for other mechanisms that could account for the clearing, such as additional bodies at $\sim 100 \mathrm{AU}$. Stringent limits are available (Casassus et al. 2013c), $\sim 4 M_{\text {jup }}$ bodies should have been detected unless they are obscured.

An interesting feature of the Cycle $0 \mathrm{HCO}^{+}(4-3)$ data is the faint filament crossing the cavity to the east, at low velocites, with perhaps another filament to the west (see Figure 10 for a summary, Casassus et al. 2013c). It is tempting to associate these $\mathrm{HCO}^{+}$features with planet-induced accretion flows, i.e., gaseous streamers due to obscured protoplanets inside the cavity, that feed the faster flows at the disk breaking radius. As the critical density for the excitation of $\mathrm{HCO}^{+}(4-3)$ is $n_{\mathrm{H}_{2}} \sim 10^{6} \mathrm{~cm}^{-3}$, and higher than the $\mathrm{CO}(3-2)$ critical density of $\sim 10^{3} \mathrm{~cm}^{-3}$, in the streamer interpretation the $\mathrm{HCO}^{+}$ filaments and high-velocity flows would trace the densest regions of the general accretion flow across the gap, which is also reflected in the "twisted" $\mathrm{CO}$ kinematics. If the free-falling accretion is initially fed by planet accretion at $\sim 100 \mathrm{AU}$, there should be corresponding azimuthal modulations of the $\mathrm{H}_{2}$ density field. Perhaps this is reflected in the structure of the faint, low-velocity $\mathrm{HCO}^{+}(4-3)$ inside the cavity.

An alternative to azimuthal modulations of the $\mathrm{H}_{2}$ density field in the cavity, is that only the abundance of $\mathrm{HCO}^{+}$is modulated. The tilted inner disk is shadowing the UV radiation required to produce $\mathrm{HCO}^{+}$in the cavity, resulting in $\mathrm{HCO}^{+}$ filaments with wide opening angles, as opposed to the thin protoplanetary streamers predicted in hydrodynamic simulations. A problem with this interpretation is that the outer ring is fairly round in $\mathrm{HCO}^{+}$, there are no obvious counterparts in $\mathrm{HCO}^{+}$of the scattered-light shadows. Perhaps this reflects a different production chain for $\mathrm{HCO}^{+}$in the outer disk, where it could be driven by charge-exchange with cosmic-ray induced $\mathrm{H}_{3}^{+}$.

\section{CONCLUSION}

The new $\mathrm{CO}(6-5)$ data, along with the orientation of the disk inferred from the scattered light shadows, have allowed us to understand the intra-cavity kinematics in HD 142527. Stellocentric accretion, starting from the outer disk and reaching close to free-fall velocities, with a steady-state mass flow fixed at the observed stellar accretion, is consistent with the bulk properties of the available $\mathrm{CO}$ isotopologue data. The observed $\mathrm{HCO}^{+}$flows are also consistent with this stellocentric accretion, but with an emissivity that is somehow modulated in azimuth. Fine structure in $\mathrm{CO}(6-5)$ also suggests non-axial symmetry inside the cavity.

While the data are consistent with a continous, yet abrupt and fast warp linking the two non-coplanar disks, further observations are required to understand the detailed structure of the intra-cavity kinematics and the inner warp. For instance, the large relative inclination observed between the inner and outer disks suggests that the fast accretion could be due to disk tearing. If the low-mass companion is contained in the inner disk and is breaking the disks, then its orbit would also be highly inclined with respect to the outer massive disk. The companion may then undergo Kozai oscillations, with higheccentricity periods that may perhaps explain the large cavity.

We acknowledge useful discussions with Sylvestre Lacour, Giuseppe Lodato, James Owen, and Daniel Price. We thank the referee for a positive report and constructive comments. This paper makes use of the following ALMA data: ALMA\# 2011.0.00465.S. ALMA is a partnership of ESO, NSF, NINS, NRC, NSC, ASIAA. The Joint ALMA Observatory is operated by ESO, AUI/NRAO, and NAOJ. Financial support was provided by Millennium Nucleus RC130007 (Chilean Ministry of Economy) and additionally by FONDECYT grants 1130949, 1141175, 3140601, 3140634, 3140393. S.M. acknowledges CONICYT-PCHA / Magister Nacional / 2014-22140628. P.R. and V.M. acknowledge support from CONICYT-ALMA grant ALMA-CONICYT 31120006. A.D. acknowledges CONICYT-ALMA grant 31120007. P.J.A. acknowledges support from NSF award AST 1313021. M.M. acknowledges CONICYT-Gemini grant 32130007. This work was partially supported by the Chilean supercomputing infrastructure of the NLHPC (ECM-02).

Facility: ALMA. 


\section{APPENDIX A OBSERVATIONS}

\section{A.1. ALMA CO(6-5) Data}

Details on the instrumental setup are described in a companion article on the continuum emission (Casassus et al. 2015). We used self-calibration to improve the dynamic range of the continuum images. Applying the same gain corrections to the line data also resulted in an improved dynamic range. Before self calibration, the peak signal in the systemic velocity channel reached $0.42 \mathrm{Jy} \mathrm{beam}^{-1}$, in natural weights (beam of $0.30 \times 0.25$ ), with a noise level of $0.08 \mathrm{Jy}$ beam $^{-1}$, which was clearly dominated by systematics rather than thermal noise. After self calibration, the peak signal increased to $0.67 \mathrm{Jy} \mathrm{beam}^{-1}$, while the rms noise level decreased to $0.04 \mathrm{Jy}_{\text {beam }}{ }^{-1}$.

Continuum subtraction under $\mathrm{CO}(6-5)$ was performed with a first-order fit to the continuum in the visibility domain. The subset of channels neighboring the line was then split off into another datafile and subsequently re-sampled in frequency into the LSRK frame.

The $\mathrm{CO}(6-5)$ data set is presented in channel maps in Figures 11 and 12, with three-channel bins. We chose to present both the restored image and the underlying MEM model.

\section{A.2. Alignment}

The comparison of the band 9 and band 7 continua reveals a positional offset of $\sim 0.07$ arcsec essentially in the east-west direction (precisely $[-0.063,-0.016]$ in arcsec, along R.A. and decl.). As detailed in a companion article devoted to a multifrequency analysis of the continuum, including a detailed presentation of the band 9 continuum, this positional offset is measured by cross-correlating the visibilities, but it is also noticeable by visual inspection of the continuum maps (for instance, by blinking the images). With the 0.35 arcsec resolution provided by these Cycle 0 observations in band 7 , the center of symmetry of the $\mathrm{CO}(3-2)$ velocity centroids coincide fairly well with the position of the central star at the epoch of observations. Hence for the multi-frequency continuum studies we chose to take the band 7 astrometry as reference.

However, at 0.02 arcsec resolutions the center of symmetry of the velocity centroid appears to be offset from the nominal stellar position as given by the default band 7 worldcoordinate-system (WCS). This represents an opportunity to improve the astrometry by tying the stellar position with the center of symmetry. We aligned the velocity centroids by applying an offset of $[-0.109,-0.031]$ arcsec to the default band 9 WCS. As illustrated in Figures 1, 8 and 13, the center of symmetry of the velocity centroid coincides with the origin of coordinates, located at the stellar position. The peak velocity dispersion is also close to the origin, but in this case the exact location of the peak depends on the moment extraction algorithm because the signal is spread over a large range in velocity and includes low signal-to-noise channels.

\section{APPENDIX B IMAGE SYNTHESIS}

\section{B.1. Angular Resolution and Statistics of the Deconvolved MEM Models}

Details on our image synthesis algorithm uvmem are given in a companion paper on the continuum emission (Casassus et al. 2015). Here we exploit the finer angular resolutions provided by the MEM models, which requires an assessment of their statistical properties.

We start by scaling the observed visibility weights so that they correspond to the observed scatter. In order to preserve the relative values of the calibrated weights, a constant scale factor is applied to all visibilities. This factor is the median of the ratio between the original weights and the dispersion values.

Estimates of the angular resolution of the deconvolved images were obtained as follows. We selected a reference channel, at the systemic velocity and split off the corresponding visibilities into a reference visibility data set. We then estimated the PSF by simulating interferometer observations on a point source, with an identical $u, v$ coverage as the reference visibilities, and with the addition of Gaussian noise, with a dispersion given by the visibility weights. In practice, the point source is a spike, defined on the same canvas as used for the deconvolved images, and whose flux density corresponds to that of the whole observed image. A deconvolved model of these simulated point-source visibilities is then obtained using the same algorithm as for the observations (i.e., uvmem). This deconvolved model represents the PSF of the deconvolution algorithm, with a central source and secondary maxima fainter by a factor of at least 20 . An elliptical Gaussian fit to the central peak defines the finest resolution achievable in the deconvolved images, of 0 ." $062 \times 0$ ". 051 .

Since the maximum-entropy regularization is nonlinear, the uvmem PSF depends on the model. We approximate the case of extended emission by spreading the total flux in a collection of spikes. The "clean" beam in this case varies by up to $50 \%$. So, for the purpose of comparing synthetic radiative transfer predictions with the uvmem model images, we chose a representative resolution of $30 \%$ times coarser than the finest PSF.

Monte-Carlo simulations provide estimates of the uncertainties associated with these MEM model datacubes. We added Gaussian noise to the observed visibilities, as given by the visibility weights, and obtained a deconvolved model image with uvmem, with the same settings as for the observations. We then took statistics on 120 realizations, checking that the average datacube satisfactorily converges on the original model datacube without the addition of noise.

\section{B.2. Moment Maps}

In the case of noisy data, the standard approach to extract moment maps by collapsing the velocity axis injects unnecessary noise when the line-of-sight line profiles are much narrower than the spatially integrated line. Rather than collapse the datacubes with standard moments, we extracted moments by restricting the velocity range above a given threshold (which is set to 0 for the restored maps, with thermal noise). For each line of sight, we identify the position of the peak specific intensity. We then search for the first nulls, both toward the red and blue, and extract a spectrum leaving room for a few more channels (two in this case). Last, we take standard moments over this reduced velocity range, and also fit a Gaussian. The resulting moment maps are embellished by median-smoothing with a square $5 \times 5$ kernel.

In the case of the MEM deconvolved models, the strict positivity requirement forces an intensity floor $I_{\min }$, which we take at $1 / 100$ the estimate noise level (in natural weights). 


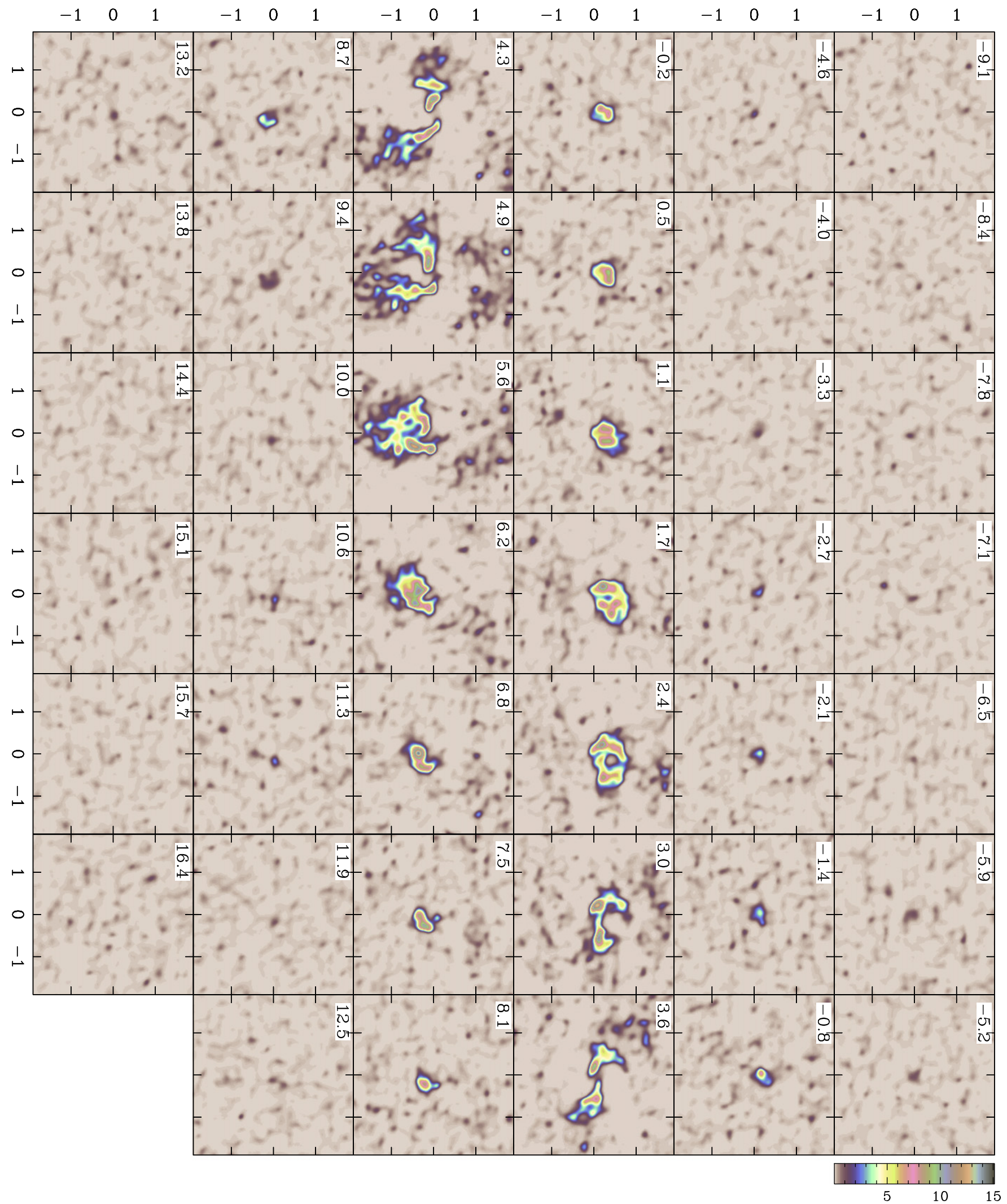

Figure 11. Channel maps in the uvmem model datacube for $\mathrm{CO}(6-5)$. We binned the datacube into three-channel averages.

We subtracted a posteriori $I_{\min }$ from the deconvolved maps. However, the positivity requirement biases the noise, so that velocity dispersions are artificially broad in regions of low signal (such as the outer ring in HD 142527 in $\mathrm{CO}(6-5))$. To account for this, we applied the velocity restrictions described above, with a threshold defined by the rms dispersion of the noise in velocity channels devoid of signal. 
The Astrophysical Journal, 811:92 (14pp), 2015 October 1

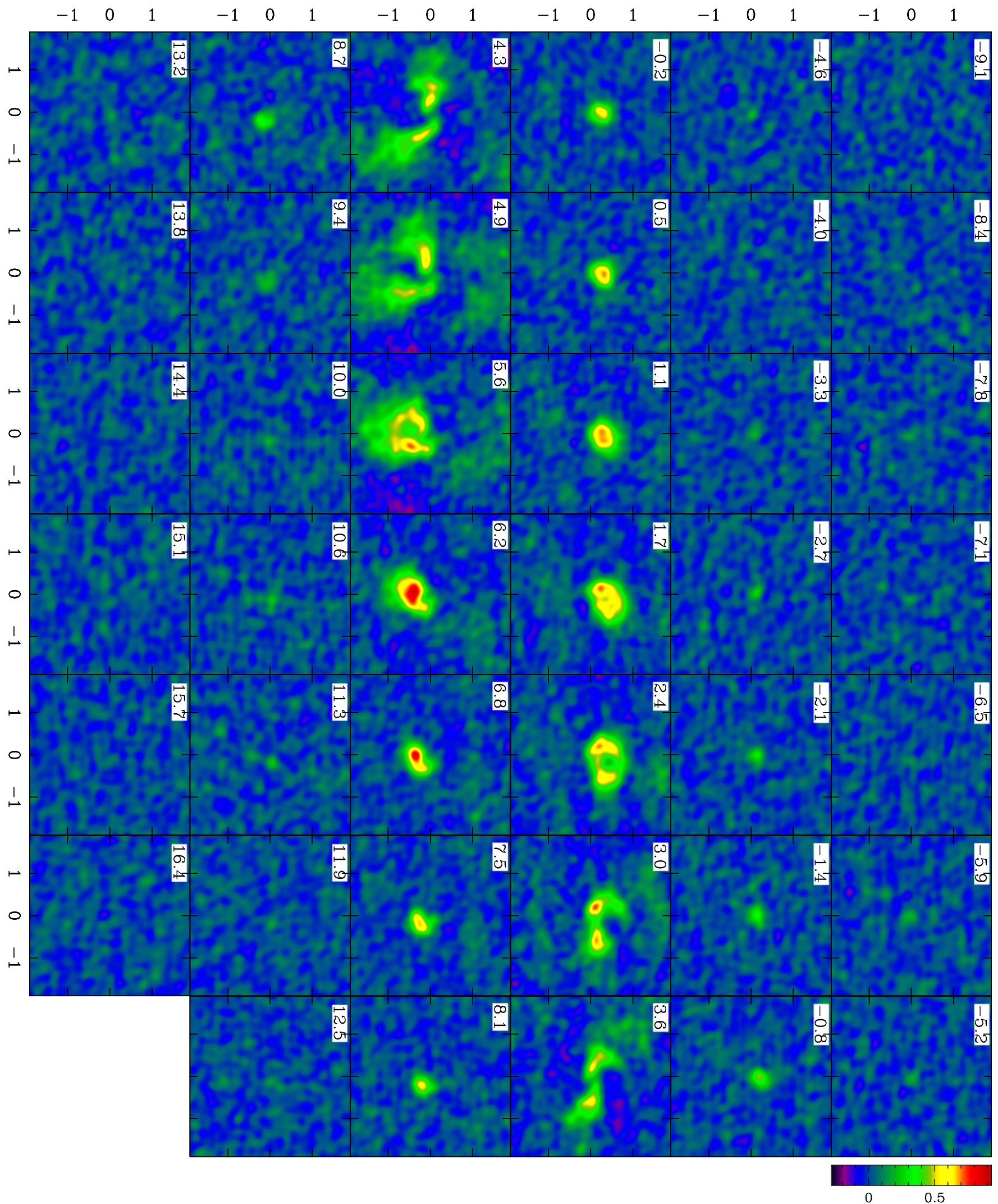

Figure 12. Channel maps in the restored $\mathrm{CO}(6-5)$ datacube. We binned the datacube into three-channel averages.

\section{B.3. Comparison of Different Image Synthesis Schemes}

The moments maps extracted on the clean, and uvmem datacubes are compared in Figure 13. The details of the centroid map do not depend on the reconstruction. However, the intensity and velocity dispersion maps inside the central beam (so within 0 "! 2 of the star) result from the superposition of several velocity components. 

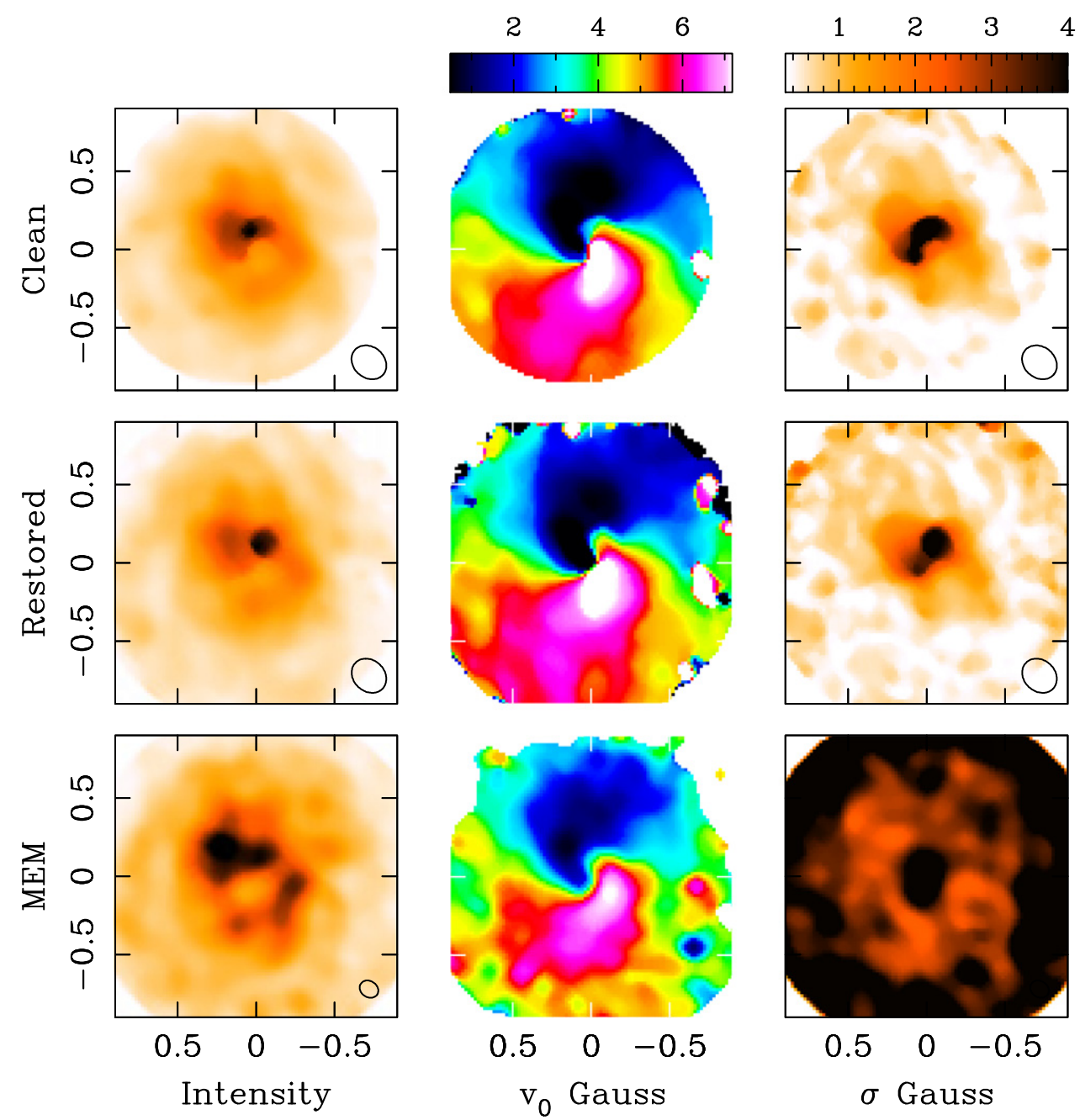

Figure 13. Moment maps in $\mathrm{CO}(6-5)$ emission. $x$ and $y$ axes show an angular offset along R.A. and decl. from the stellar position. The upper row shows maps extracted on the clean datacubes, produced in Briggs weights. The middle and bottom rows, respectively, show the maps extracted on the restored uvmem+CASA datacubes, in Briggs weights, and the deconvolved MEM models. Columns correspond to intensity (left), velocity centroid (middle), and $1 \sigma$ velocity dispersion (right). The moments from the deconvolved MEM maps are extracted without the thresholds (Appendix B.2) used in Figure 1.
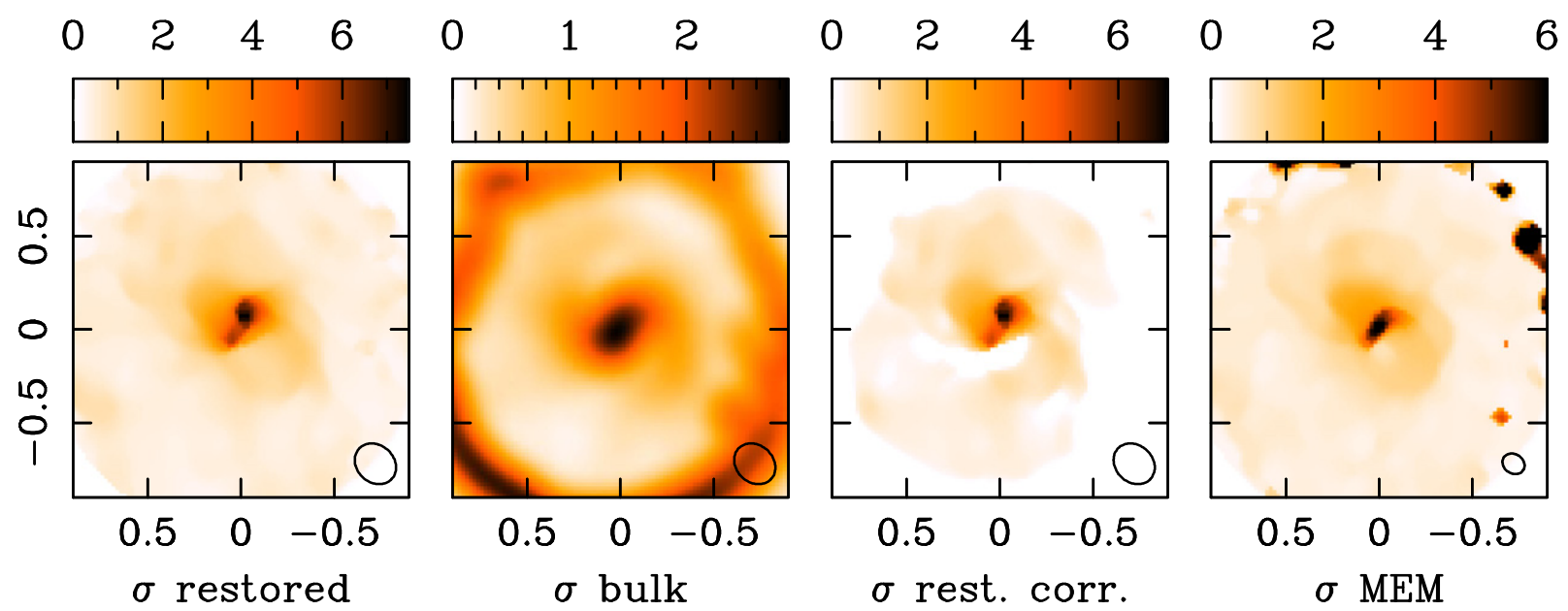

Figure 14. Intrinsic line width and structure within the central beam. All images are plotted in linear stretch. From left to right we show the restored velocity dispersion in Briggs weights, $\sigma_{\text {rest. }}$, the bulk flow computed from the MEM centroid, $\sigma_{\text {bulk }}$, the intrinsic line width $\sigma_{\text {int. }}$ (labeled " $\sigma$ rest. corr."), and the deconvolved line width $\sigma_{\text {mem }}$. 


\section{APPENDIX C IMPACT OF A FINITE BEAM IN ESTIMATES OF INTRINSIC LINE WIDTHS}

An alternative cross check on the quality of the deconvolved velocity dispersion as an estimate of the intrinsic line width, at least away from the central beam, is to take statistics on the velocity centroid $v_{\text {mem }}$ extracted on the MEM datacubes. We degrade $v_{\text {mem }}$ with the same beam that was used in the Briggs restoration, $v_{\text {mem }}^{s} \equiv\left\langle v_{\text {mem }}\right\rangle=v_{\text {mem }} \otimes B$, and calculate the velocity dispersion in the restored maps due to the bulk flow with $\sigma_{\text {bulk }}=\sqrt{\left\langle v_{\text {mem }}^{2}\right\rangle-\left(v_{\text {mem }}^{s}\right)^{2}}$, where $\left\langle v_{\text {mem }}^{2}\right\rangle \equiv v_{\text {mem }}^{2} \otimes B$. We then subtract $\sigma_{\text {bulk }}$ in quadrature to estimate the intrinsic line width: $\sigma_{\text {int. }}=\sqrt{\sigma_{\text {rest. }}-\sigma_{\text {bulk }}}$, where $\sigma_{\text {rest. }}$ is the $1 \sigma$ velocity dispersion extracted from the restored datacubes. The corresponding dispersion at $\boldsymbol{P}$ is $\sigma_{\text {int. }}(\boldsymbol{P})=1.81 \mathrm{~km} \mathrm{~s}^{-1}$. A few pixels have slightly lower $\sigma_{\text {rest. }}$ than $\sigma_{\text {bulk }}$, which may stem from the finite resolution of $v_{\mathrm{mem}}$, or to non-Gaussian line profiles. Because these pixels represent a small fraction, we set them to zero. Figure 14 compares the deconvolved velocity dispersion, also shown in Figure 1, with the dispersion of the MEM datacube after correction for bulk motion.

The $\sigma_{\text {rest. }}$ map in Figure 14 shows interesting structure on scales much smaller than the clean beam. Two peaks in velocity dispersion are very reminiscent of the orientation and separation between HD 142527 and HD 142527B (Biller et al. 2012; Close et al. 2014). The binary separation is 84 mas along $\mathrm{PA}=127^{\circ}$, and the mass ratio is $<0.1$, where the inequality is due to the correction for accretion luminosity on HD 142527B. Although it is tempting to associate the secondary peak in $\mathrm{CO}(6-5)$ velocity dispersion with $\mathrm{HD}$ $142527 \mathrm{~B}$, we see that it does not persist in the deconvolved maps, while the main peak also shifts slightly in position. These peaks must reflect features of the underlying velocity field, so that the corresponding pixels mix blue- and redshifted emission in a coarse beam.

\section{REFERENCES}

Avenhaus, H., Quanz, S. P., Schmid, H. M., et al. 2014, ApJ, 781, 87 Bate, M. R., Lodato, G., \& Pringle, J. E. 2010, MNRAS, 401, 1505 Biller, B., Lacour, S., Juhász, A., et al. 2012, ApJL, 753, L38

Canovas, H., Ménard, F., Hales, A., et al. 2013, A\&A, 556, A123

Casassus, S., Hales, A., de Gregorio, I., et al. 2013a, A\&A, 553, A64

Casassus, S., Perez, M. S., Jordán, A., et al. 2012, ApJL, 754, L31

Casassus, S., Perez, S. M., van der Plas, G., et al. 2013b, in NRAO workshop, Transformational Science with ALMA: From Dust to Rocks to Planets Casassus, S., van der Plas, G., Perez, S. M., et al. 2013c, Natur, 493, 191

Casassus, S., Wright, C., Marino, S., et al. 2015, ApJ, submitted

Christiaens, V., Casassus, S., Perez, S., van der Plas, G., \& Ménard, F. 2014, ApJL, 785, L12

Close, L. M., Follette, K. B., Males, J. R., et al. 2014, ApJL, 781, L30

Dodson-Robinson, S. E., \& Salyk, C. 2011, ApJ, 738, 131

Doğan, S., Nixon, C., King, A., \& Price, D. J. 2015, MNRAS, 449, 1251

Dullemond, C., Juhasz, A., Pohl, A., et al. 2015, RADMC3D v0.39 http:// www.ita.uni-heidelberg.de/dullemond/software/radmc-3d/

Facchini, S., Lodato, G., \& Price, D. J. 2013, MNRAS, 433, 2142

Facchini, S., Ricci, L., \& Lodato, G. 2014, MNRAS, 442, 3700

Fujiwara, H., Honda, M., Kataza, H., et al. 2006, ApJL, 644, L133

Fukagawa, M., Tamura, M., Itoh, Y., et al. 2006, ApJL, 636, L153

Fukagawa, M., Tsukagoshi, T., Momose, M., et al. 2013, PASJ, 65, L14

Garcia Lopez, R., Natta, A., Testi, L., \& Habart, E. 2006, A\&A, 459, 837

Hughes, A. M., Andrews, S. M., Espaillat, C., et al. 2009, ApJ, 698, 131

Marino, S., Perez, S., \& Casassus, S. 2015, ApJL, 798, L44

Miranda, R., \& Lai, D. 2015, arXiv:1504.02917

Nealon, R., Price, D. J., \& Nixon, C. J. 2015, MNRAS, 448, 1526

Nixon, C., King, A., \& Price, D. 2013, MNRAS, 434, 1946

Nixon, C., King, A., Price, D., \& Frank, J. 2012, ApJL, 757, L24

Perez, S., Casassus, S., Ménard, F., et al. 2015, ApJ, 798, 85

Pontoppidan, K. M., Blake, G. A., \& Smette, A. 2011, ApJ, 733, 84

Pringle, J. E. 1992, MNRAS, 258, 811

Rosenfeld, K. A., Chiang, E., \& Andrews, S. M. 2014, ApJ, 782, 62

Stahl, O., Casassus, S., \& Wilson, T. 2008, A\&A, 477, 865

Teague, R., Semenov, D., Guilloteau, S., et al. 2015, A\&A, 574, A137

Teyssandier, J., Terquem, C., \& Papaloizou, J. C. B. 2013, MNRAS, 428, 658

van der Plas, G., Casassus, S., Ménard, F., et al. 2014, ApJL, 792, L25

Wilson, T. L., \& Rood, R. 1994, ARA\&A, 32, 191

Wootten, A., Snell, R., \& Glassgold, A. E. 1979, ApJ, 234, 876

Zhu, Z., Nelson, R. P., Hartmann, L., Espaillat, C., \& Calvet, N. 2011, ApJ, 729, 47 